\title{
Probabilities of hitting a convex hull
}

\author{
Zhenxia Liu and Xiangfeng Yang
}

\section{Linköping University Post Print}

\section{Tweet}

N.B.: When citing this work, cite the original article.

Original Publication:

Zhenxia Liu and Xiangfeng Yang, Probabilities of hitting a convex hull, 2014, Comptes rendus. Mathematique, (352), 11, 935-940.

http://dx.doi.org/10.1016/j.crma.2014.08.015

Copyright: Elsevier Masson

http://www.elsevier-masson.fr/

Postprint available at: Linköping University Electronic Press

http://urn.kb.se/resolve?urn=urn:nbn:se:liu:diva-112620 


\title{
Probabilities of hitting a convex hull Probabilités d'atteinte d'une envelopple convexe
}

\author{
Zhenxia Liu* Xiangfeng Yang ${ }^{\dagger}$
}

August 27, 2014

\begin{abstract}
In this note, we consider the non-negative least square method with a random matrix. This problem has connections with the probability that the origin is not in the convex hull of many random points. As related problems, suitable estimates are obtained as well on the probability that a small ball does not hit the convex hull.
\end{abstract}

\begin{abstract}
Dans cette Note nous appliquons la méthode des moindres carrés non-négatifs avec une matrice aléatoire. Ce problème est connecté à la probabilité que l'enveloppe convexe de points aléatoires ne contienne pas l'origine. En relation avec ce problème nous obtenons aussi des estimations de la probabilité qu'une petite boule ne rencontre pas une enveloppe convexe.
\end{abstract}

Keywords and phrases: Convex hull, uniform distribution, non-negative least square method AMS 2010 subject classifications: 60D05, 52A22

\section{Introduction}

Let $n$ and $m$ be two positive integers with $n \leq m$. Suppose that $A$ is a $n \times m$ matrix and $\mathbf{b}$ is a vector in $\mathbb{R}^{n}$. In mathematical optimization and other research fields, it is frequent to consider the non-negative least square solution to a linear system $A \mathbf{X}=\mathbf{b}$ with $\mathbf{X}=\left(x_{1}, x_{2}, \ldots, x_{m}\right)^{T} \in \mathbb{R}^{m}$ under the constraint $\min _{1 \leq i \leq m} x_{i} \geq 0$. The non-negativity constraints occur naturally in various models involving non-negative data; see [1], [3] and [7]. More generally for non-negative random designs, the matrix $A$ is assumed to be random; see [4] and references therein for this aspect.

The first topic of this note is to investigate the probability $\mathbb{P}\left\{A \mathbf{X}=\mathbf{b}, \min _{1 \leq i \leq m} x_{i} \geq 0\right\}$ when $A$ is a random matrix with suitable restrictions; see Theorem 2.1. The idea of the proof is to change this probability to the one involving the event that the origin is not in the convex hull of many random points, and then apply a well-known result by Wendel [11]. However, instead of applying Wendel's result directly, we propose a new probabilistic proof of it. This probabilistic proof allows us to work on a more general probability of hitting a convex hull by a small ball (instead of the origin) in $\mathbb{R}^{n}$; see Theorem 4.1.

${ }^{*}$ zhenxia.liu@hotmail.com, Blåeldsvägen 12B, Sturefors, Sweden

†xiangfeng.yang@liu.se, Department of Mathematics, Linköping University, SE-581 83 Linköping, Sweden 
The study on random convex hulls dates back to 1960s from various perspectives. For instance, in [10] and [2] the expected perimeter of a random convex hull was derived. The expected number of edges of a random convex hull was obtained in [8]. For expected area or volume of a random convex hull, we refer to [5]. As mentioned earlier, in [11] the probability that the origin does not belong to a random convex hull was perfectly established. In Section 3, we derive an explicit form for the probability that a ball with a small radius $\delta$ in $\mathbb{R}^{2}$ does not belong to the convex hull of many i.i.d. random points; see Theorem 3.1. This type of probability was considered in [6] together with circle coverage problems. Because of addition assumptions there, unfortunately the results (Corollary 4.2 and Example 4.1) in [6] cannot recover our result Theorem 3.1 in this note. A more detailed survey on random convex hulls is included in [9].

\section{A linear system with a random matrix}

Since the one-dimension $n=1$ is trivial, we consider higher dimensions $n \geq 2$. In the proof of the next result, a connection is established between the probabilities of hitting a convex hull and the non-negative solutions to a linear system.

Theorem 2.1. Let $A$ be an $n \times m, 2 \leq n \leq m$, matrix such that the entries are independent nonnegative continuous random variables. Suppose that these random variables have the same mean $\mu$, and are symmetric about the mean. Then the linear system $A \mathbf{X}=(1,1, \ldots, 1)^{T}$ has a non-negative solution with probability

$$
1-2^{-m+1} \sum_{k=0}^{n-2}\left(\begin{array}{c}
m-1 \\
k
\end{array}\right) .
$$

When $m=n$, it simplifies to $2^{-n+1}$.

Proof. We set the entries of $A$ as $\left\{a_{i j}\right\}$, then $\sum_{j=1}^{m} a_{i j} x_{j}=1$ for $1 \leq i \leq n$. Summing on $i$, we obtain $\sum_{j=1}^{m}\left(\sum_{i=1}^{n} a_{i j}\right) x_{j}=n$. Let $c_{j}=\frac{1}{n} \sum_{i=1}^{n} a_{i j}$, then $\sum_{j=1}^{m} c_{j} x_{j}=1$. Thus, we can rewrite the linear system $\sum_{j=1}^{m} a_{i j} x_{j}=1$ as $\sum_{j=1}^{m}\left(a_{i j}-c_{j}\right) x_{j}=0$. Let $\mathbf{a}_{1}, \ldots, \mathbf{a}_{m}$ be the column vectors of $A$, and $\mathbf{v}=(1,1, \ldots, 1)^{T}$. If we denote $\mathbf{w}_{j}=\mathbf{a}_{j}-c_{j} \mathbf{v}$, Then the linear system $\sum_{j=1}^{m} a_{i j} x_{j}=1$ for $1 \leq i \leq n$ has a non-negative solution if and only if there exist $x_{1}, x_{2}, \ldots, x_{m} \geq 0$ with $x_{1}+x_{2}+\ldots+x_{m}>0$ such that $\sum_{j=1}^{m} x_{j} \mathbf{w}_{j}=\mathbf{0}$. In other words, the origin $\mathbf{0}$ belongs to the convex hull of $\left\{\mathbf{w}_{1}, \mathbf{w}_{2}, \ldots, \mathbf{w}_{m}\right\}$. We show that $\left\{\mathbf{w}_{j}\right\}$ are symmetric. Indeed,

$$
\begin{aligned}
& \mathbb{P}\left\{\mathbf{w}_{j}>\left(t_{1}, t_{2}, \ldots, t_{n}\right)^{T}\right\} \\
& =\mathbb{P}\left\{a_{i j}-\frac{1}{n} \sum_{k=1}^{n} a_{k j}>t_{i}, 1 \leq i \leq n\right\} \\
& =\mathbb{P}\left\{\frac{1}{n} \sum_{k=1}^{n}\left(a_{i j}-a_{k j}\right)>t_{i}, 1 \leq i \leq n\right\} \\
& =\mathbb{P}\left\{\frac{1}{n} \sum_{k=1}^{n}\left[\left(\mu-a_{i j}\right)-\left(\mu-a_{k j}\right)\right]>t_{i}, 1 \leq i \leq n\right\} \\
& =\mathbb{P}\left\{-\frac{1}{n} \sum_{k=1}^{n}\left(a_{i j}-a_{k j}\right)>t_{i}, 1 \leq i \leq n\right\}=\mathbb{P}\left\{-w_{j}>\left(t_{1}, t_{2}, \ldots, t_{n}\right)^{T}\right\} .
\end{aligned}
$$


Clearly, $\left\{\mathbf{w}_{j}\right\}$ are random vectors in $\mathbb{R}^{n}$ that lie on the hyperplane $L=\left\{\left(y_{1}, y_{2}, \ldots, y_{n}\right) \in \mathbb{R}^{n}\right.$ : $\left.y_{1}+y_{2}+\ldots+y_{n}=0\right\}$. Let $p(k, m)$ be the probability that $\mathbf{0}$ does not belong to the convex hull of $m$ symmetric random vectors in $\mathbb{R}^{n}$ that lie on a $k$-dimensional subspace of $\mathbb{R}^{n}$. We now compute the probability $p(n-1, m)$. The method below is a probability version of a geometric argument of Wendel [11]. Let $h$ be the indicator function of the event $\mathbf{0} \notin \operatorname{conv}\left(\mathbf{w}_{1}, \mathbf{w}_{2}, \ldots, \mathbf{w}_{m}\right)$. That is, $h\left(\mathbf{w}_{1}, \mathbf{w}_{2}, \ldots, \mathbf{w}_{m}\right)=1$ if there exists a non-zero vector $\mathbf{b}$ such that $\left\langle\mathbf{w}_{i}, \mathbf{b}\right\rangle \geq 0$ for all $1 \leq i \leq m$, and $h\left(\mathbf{w}_{1}, \mathbf{w}_{2}, \ldots, \mathbf{w}_{m}\right)=0$ otherwise. Then,

$$
p(n-1, m)=\mathbb{P}\left\{\mathbf{0} \notin \operatorname{conv}\left(\mathbf{w}_{1}, \mathbf{w}_{2}, \ldots, \mathbf{w}_{m}\right)\right\}=\mathbb{E}_{\mathbf{w}} h\left(\mathbf{w}_{1}, \mathbf{w}_{2}, \ldots, \mathbf{w}_{m}\right) .
$$

Because $\left\{\mathbf{w}_{i}\right\}$ are symmetric, if we let $\left\{\varepsilon_{i}\right\}$ be i.i.d. Bernoulli random variables, then

$$
p(n-1, m)=\mathbb{E}_{\varepsilon} \mathbb{E}_{\mathbf{w}} h\left(\varepsilon_{1} \mathbf{w}_{1}, \varepsilon_{2} \mathbf{w}_{2}, \ldots, \varepsilon_{m} \mathbf{w}_{m}\right) .
$$

Noticing that conditioning on $\varepsilon^{\prime}=\left(\varepsilon_{1}, \varepsilon_{2}, \ldots, \varepsilon_{m-1}\right)$, we have

$$
\begin{aligned}
p(n-1, m)= & \mathbb{E}_{\varepsilon^{\prime}} \mathbb{E}_{\mathbf{w}} \mathbb{E}_{\varepsilon m} h\left(\varepsilon_{1} \mathbf{w}_{1}, \varepsilon_{2} \mathbf{w}_{2}, \ldots, \varepsilon_{m} \mathbf{w}_{m}\right) \\
= & \frac{1}{2} \mathbb{E}_{\varepsilon^{\prime}} \mathbb{E}_{\mathbf{w}} \mathbb{E}_{\varepsilon m} h\left(\varepsilon_{1} \mathbf{w}_{1}, \varepsilon_{2} \mathbf{w}_{2}, \ldots, \varepsilon_{m-1} \mathbf{w}_{m-1}\right) \\
& +\frac{1}{2} \mathbb{E}_{\varepsilon^{\prime}} \mathbb{E}_{\mathbf{w}}\left[2 \mathbb{E}_{\varepsilon m} h\left(\varepsilon_{1} \mathbf{w}_{1}, \varepsilon_{2} \mathbf{w}_{2}, \ldots, \varepsilon_{m} \mathbf{w}_{m}\right)-h\left(\varepsilon_{1} \mathbf{w}_{1}, \varepsilon_{2} \mathbf{w}_{2}, \ldots, \varepsilon_{m-1} \mathbf{w}_{m-1}\right)\right] \\
= & \frac{1}{2} p(n-1, m-1)+\frac{1}{2} \mathbb{E}_{\varepsilon^{\prime}} \mathbb{E}_{\mathbf{w}} R
\end{aligned}
$$

where

$$
R:=h\left(\varepsilon_{1} \mathbf{w}_{1}, \varepsilon_{2} \mathbf{w}_{2}, \ldots, \mathbf{w}_{m}\right)+h\left(\varepsilon_{1} \mathbf{w}_{1}, \varepsilon_{2} \mathbf{w}_{2}, \ldots,-\mathbf{w}_{m}\right)-h\left(\varepsilon_{1} \mathbf{w}_{1}, \varepsilon_{2} \mathbf{w}_{2}, \ldots, \varepsilon_{m-1} \mathbf{w}_{m-1}\right) .
$$

We see that $R \in\{0,1\}$, and $R=1$ if and only if

$$
h\left(\varepsilon_{1} \mathbf{w}_{1}, \varepsilon_{2} \mathbf{w}_{2}, \ldots, \mathbf{w}_{m}\right)=h\left(\varepsilon_{1} \mathbf{w}_{1}, \varepsilon_{2} \mathbf{w}_{2}, \ldots,-\mathbf{w}_{m}\right)=1 .
$$

That is, there exists vectors $\mathbf{b}_{1}, \mathbf{b}_{2}$ such that $\left\langle\varepsilon_{i} \mathbf{w}_{i}, \mathbf{b}_{1}\right\rangle \geq 0,\left\langle\varepsilon_{i} \mathbf{w}_{i}, \mathbf{b}_{2}\right\rangle \geq 0$ for $1 \leq i \leq m-1$ and $\left\langle\mathbf{w}_{m}, \mathbf{b}_{1}\right\rangle \geq 0,\left\langle\mathbf{w}_{m}, \mathbf{b}_{2}\right\rangle \leq 0$. Thus we can find $\alpha, \beta>0$ such that for $\mathbf{c}=\alpha \mathbf{b}_{1}+\beta \mathbf{b}_{2}$, we have $\left\langle\varepsilon_{i} \mathbf{w}_{i}, \mathbf{c}\right\rangle \geq 0$ for $1 \leq i \leq m-1$, and $\left\langle\mathbf{w}_{m}, \mathbf{c}\right\rangle=0$. On the other hand, if we can find such a vector $\mathbf{c}$, then of course $h\left(\varepsilon_{1} \mathbf{w}_{1}, \varepsilon_{2} \mathbf{w}_{2}, \ldots, \mathbf{w}_{m}\right)=h\left(\varepsilon_{1} \mathbf{w}_{1}, \varepsilon_{2} \mathbf{w}_{2}, \ldots,-\mathbf{w}_{m}\right)=1$. Therefore, $R=1$ if and only if there exists a vector $\mathbf{c}$ such that $\mathbf{c} \perp \mathbf{w}_{m}$ such that $\left\langle\varepsilon_{i} \mathbf{w}_{i}, \mathbf{c}\right\rangle \geq 0$ for $1 \leq i \leq m-1$. If we let $\mathbf{u}_{i}$ be the orthogonal projection of $\mathbf{w}_{i}$ on to $\mathbf{w}_{m}^{\perp}$ for $1 \leq i \leq m-1$, then $R=1$ if and only if $h\left(\varepsilon_{1} \mathbf{u}_{1}, \varepsilon_{2} \mathbf{u}_{2}, \ldots, \varepsilon_{m-1} \mathbf{u}_{m-1}\right)=1$. From the fact that $\left\{\mathbf{u}_{i}\right\}$ are vectors in $\mathbb{R}^{n}$ that lies on the $(n-2)$-dimensional subspace $\mathbf{w}_{m}^{\perp} \cap L$, it follows that

$$
\mathbb{E}_{\mathcal{\varepsilon}^{\prime}} \mathbb{E}_{\mathbf{w}} R=\mathbb{E}_{\mathcal{\varepsilon}^{\prime}} \mathbb{E}_{\mathbf{u}} h\left(\varepsilon_{1} \mathbf{u}_{1}, \varepsilon_{2} \mathbf{u}_{2}, \ldots, \varepsilon_{m-1} \mathbf{u}_{m-1}\right)=p(n-2, m-1) .
$$

Hence, we obtain the identity

$$
p(n-1, m)=\frac{1}{2} p(n-1, m-1)+\frac{1}{2} p(n-2, m-1)
$$

for all $m \geq n \geq 2$. Note that $p(1, k)=2^{-k+1}$ and $p(k, 1)=1$ for $k \geq 1$. By using induction and the combinatorial identity

$$
\left(\begin{array}{c}
m-2 \\
k
\end{array}\right)+\left(\begin{array}{c}
m-2 \\
k+1
\end{array}\right)=\left(\begin{array}{c}
m-1 \\
k+1
\end{array}\right)
$$


it is straightforward to check that

$$
p(n-1, m)=2^{-m+1} \sum_{k=0}^{n-2}\left(\begin{array}{c}
m-1 \\
k
\end{array}\right)
$$

for all $m \geq n \geq 2$.

\section{$3 \quad$ Probability of avoiding a small disk in $\mathbb{R}^{2}$}

Let random vectors $\left\{X_{i}\right\}_{i=1,2, \ldots, m}$ be independently and uniformly distributed in the unit ball of $\mathbb{R}^{2}$. The result in Section 2 states that the probability that the origin is not in the convex hull of $\left\{X_{i}\right\}_{i=1,2, \ldots, m}$ is $p(2, m)=m \cdot 2^{-m+1}$. In this section, our goal is to find a more general result, namely, the probability that a ball with a small radius in $\mathbb{R}^{2}$ does not belong to the convex hull of $\left\{X_{i}\right\}_{i=1,2, \ldots, m}$. We will prove the following result.

Theorem 3.1. Suppose that $\left\{X_{i}\right\}_{i=1,2, \ldots, m}$ are independently and uniformly distributed random vectors in the unit ball of $\mathbb{R}^{2}$. Let $p^{\delta}(2, m)$ denote the probability that a ball with a small radius $\delta$ in $\mathbb{R}^{2}$ does not belong to the convex hull of $\left\{X_{i}\right\}_{i=1,2, \ldots, m}$. Then

$$
p^{\delta}(2, m)=\frac{m}{2^{m-1}}\left(1-\delta^{2}\right)\left[1-\frac{2 \delta \sqrt{1-\delta^{2}}}{\pi}-\frac{2}{\pi} \sin ^{-1}(\delta)\right]^{m-1} .
$$

Proof. There are two different cases: the closest point on the convex hull of $\left\{X_{i}\right\}_{i=1,2, \ldots, m}$ to the origin is a vertex (see Case 2), and the closest point on the convex hull of $\left\{X_{i}\right\}_{i=1,2, \ldots, m}$ to the origin is not a vertex but a point on an edge (see Case 1). For each case, we compute the probability respectively.

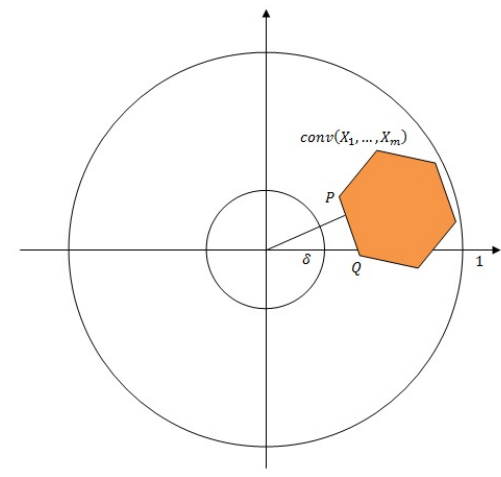

Case 1

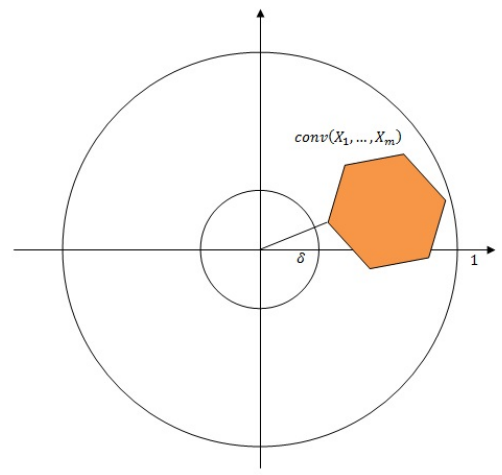

Case 2

Step 1. Let $P$ and $Q$ be two independently and uniformly distributed random points in the unit ball. We calculate the probability of the event $E(r)$ that the distance between the origin and the line segment $P Q$ is less than or equal to $r$, and the closest point to the origin is not $P$ or $Q$. Let $(\lambda, \theta)$ be the polar coordinates of $P$. Let $L$ be the line passing through $P$ and being perpendicular to $O P$. Then the line $L$ divides the unit disk into two parts, say $R_{1}$ and $R_{2}$, where $R_{2}$ is the larger 
region that contains the origin. Further, we let $D$ be the disk with $O P$ as its diameter. Then it is obvious that $D \subset R_{2}$.

If $Q \in R_{1}$, then $P$ is the closest point to the origin. If $Q \in D$, then $Q$ is the closest point to the origin. If $Q \in R_{2} \backslash D$, then the closest point of the line segment $P Q$ to the origin is not $P$ or $Q$.

If $\lambda \leq r$, then for all $Q \in R_{2} \backslash D$, the distance between the origin and the line segment $P Q$ is less than or equal to $r$; if $\lambda>r$, then to ensure that the distance between the origin and the line segment $P Q$ is less than or equal to $r$, the point $Q$ must land in the region $S$ which is between the two tangent lines from $P$ to the circle centers at the origin with radius $r$.

In conclusion, we have the following: if $\lambda \leq r$, then $Q \in R_{2} \backslash D$; if $\lambda>r$, then $Q \in S \cap\left(R_{2} \backslash D\right)$.

The set $R_{2}$ has area $\pi / 2+\int_{0}^{\lambda} 2 \sqrt{1-x^{2}} d x$, and $D$ has area $\pi \lambda^{2} / 4$. Thus $R_{2} \backslash D$ has area

$$
\pi / 2+\int_{0}^{\lambda} 2 \sqrt{1-x^{2}} d x-\pi \lambda^{2} / 4
$$

To calculate the area $F:=S \cap\left(R_{2} \backslash D\right)$, we let $T_{1}$ and $T_{2}$ be the two tangent points. The angle between the two tangent lines is $2 \sin ^{-1}(r / \lambda)$. We draw two lines through the origin which are parallel to the two tangent lines. The region $G$ that lies between these two lines and inside $F$ has area $\sin ^{-1}(r / \lambda)$. To calculate the area of the region $F \backslash G$, we connect $O$ with $T_{1}$ and $T_{2}$. Let $A$ be the area between the line segment $O T_{1}$ and the small arc $O T_{1}$ on $D$. Then the area of $F \backslash G$ is

$$
2 \int_{0}^{r} \sqrt{1-x^{2}} d x-2 A
$$

To calculate $A$, we let $M$ be the center of $D$. Then $\angle O M T_{1}=2 \sin ^{-1}(r / \lambda)$, the fan $O M T_{1}$ has area $(\lambda / 2)^{2} \sin ^{-1}(r / \lambda)$, and the $\triangle O M T_{1}$ has area $r \sqrt{\lambda^{2}-r^{2}} / 4$. Hence, the area of $F$ is

$$
\sin ^{-1}(r / \lambda)+2 \int_{0}^{r} \sqrt{1-x^{2}} d x-\frac{\lambda^{2}}{2} \sin ^{-1}(r / \lambda)+\frac{1}{2} r \sqrt{\lambda^{2}-r^{2}} .
$$

Therefore, given $P$ at $(\lambda, \theta)$, if $\lambda \leq r$, then the event $E(r)$ occurs with probability

$$
\frac{1}{2}+\frac{2}{\pi} \int_{0}^{\lambda} \sqrt{1-x^{2}} d x-\lambda^{2} / 4
$$

If $\lambda>r$, then the event $E(r)$ occurs with probability

$$
\frac{1}{\pi} \sin ^{-1}(r / \lambda)+\frac{2}{\pi} \int_{0}^{r} \sqrt{1-x^{2}} d x-\frac{\lambda^{2}}{2 \pi} \sin ^{-1}(r / \lambda)+\frac{1}{2 \pi} r \sqrt{\lambda^{2}-r^{2}} .
$$

Thus, the event $E(r)$ occurs with probability

$$
\begin{aligned}
\mathbb{P}\{E(r)\}= & \int_{0}^{r}\left(\frac{1}{2}+\frac{2}{\pi} \int_{0}^{\lambda} \sqrt{1-x^{2}} d x-\lambda^{2} / 4\right) 2 \lambda d \lambda \\
& +\int_{r}^{1}\left(\frac{1}{\pi} \sin ^{-1}(r / \lambda)+\frac{2}{\pi} \int_{0}^{r} \sqrt{1-x^{2}} d x-\frac{\lambda^{2}}{2 \pi} \sin ^{-1}(r / \lambda)+\frac{1}{2 \pi} r \sqrt{\lambda^{2}-r^{2}}\right) 2 \lambda d \lambda .
\end{aligned}
$$


This implies that

$$
\begin{aligned}
\frac{d \mathbb{P}\{E(r)\}}{d r} & \left(\frac{1}{2}+\frac{2}{\pi} \int_{0}^{r} \sqrt{1-x^{2}} d x-r^{2} / 4\right) 2 r-\left(\frac{1}{2}+\frac{2}{\pi} \int_{0}^{r} \sqrt{1-x^{2}} d x-r^{2} / 4\right) 2 r \\
& +\int_{r}^{1}\left(\frac{1}{\pi \sqrt{\lambda^{2}-r^{2}}}+\frac{2}{\pi} \sqrt{1-r^{2}}-\frac{\lambda^{2}}{2 \pi \sqrt{\lambda^{2}-r^{2}}}+\frac{1}{2 \pi} \sqrt{\lambda^{2}-r^{2}}-\frac{r^{2}}{2 \pi \sqrt{\lambda^{2}-r^{2}}}\right) 2 \lambda d \lambda \\
= & \int_{r}^{1}\left(\frac{1-r^{2}}{\pi \sqrt{\lambda^{2}-r^{2}}}+\frac{2}{\pi} \sqrt{1-r^{2}}\right) 2 \lambda d \lambda \\
= & \frac{4}{\pi}\left(1-r^{2}\right)^{3 / 2} .
\end{aligned}
$$

We note that here a particular case is $\mathbb{P}\{E(1)\}=\int_{0}^{1} \frac{4}{\pi}\left(1-r^{2}\right)^{3 / 2} d r=\frac{3}{4}$, which is the probability that the closest point is not reached at a vertex point.

Step 2. Now we calculate the probability $P(\delta)$ that the distance between the origin and the convex hull is at least $\delta$. If the closest point is a vertex of the convex hull, then it could be any of the $m$ points. Thus we need to first choose a point, say $P(r, \theta)$, and we have $m$ different choices. Let $L$ be the line passing through $P$ which is perpendicular to $O P$. Then all the other points must land on the outer side of the line $L$. The area of that region is $\int_{r}^{1} 2 \sqrt{1-x^{2}} d x$. Thus, the corresponding probability is

$$
P_{1}\{\delta\}=m \int_{\delta}^{1}\left[\frac{1}{\pi} \int_{r}^{1} 2 \sqrt{1-x^{2}} d x\right]^{m-1} 2 r d r .
$$

In particular, if $\delta=1$, then we have $P(1)=1 / 2$. In other words, with probability $1 / 4$, the closest point is a vertex.

If the closest point is not a vertex, then it is on the line segment between two vertices. Since any two vertices are equally likely, we have $m(m-1) / 2$ different choices. The probability in this case is

$$
P_{2}\{\delta\}=\frac{m(m-1)}{2} \int_{\delta}^{1}\left[\frac{1}{\pi} \int_{r}^{1} 2 \sqrt{1-x^{2}} d x\right]^{m-2} \frac{4}{\pi}\left(1-r^{2}\right)^{3 / 2} d r .
$$

Hence, the total probability is

$$
\begin{aligned}
P(\delta)= & m \int_{\delta}^{1}\left[\frac{1}{\pi} \int_{r}^{1} 2 \sqrt{1-x^{2}} d x\right]^{m-1} 2 r d r \\
& +\frac{m(m-1)}{2} \int_{\delta}^{1}\left[\frac{1}{\pi} \int_{r}^{1} 2 \sqrt{1-x^{2}} d x\right]^{m-2} \frac{4}{\pi}\left(1-r^{2}\right)^{3 / 2} d r \\
= & m\left(1-\delta^{2}\right)\left[\frac{2}{\pi} \int_{\delta}^{1} \sqrt{1-x^{2}} d x\right]^{m-1} \\
= & \frac{m}{2^{m-1}} \cdot\left(1-\delta^{2}\right)\left[1-\frac{2 \delta \sqrt{1-\delta^{2}}}{\pi}-\frac{2}{\pi} \sin ^{-1} \delta\right]^{m-1},
\end{aligned}
$$

where the second equality is from integration by parts. 


\section{Probability of avoiding a small ball in $\mathbb{R}^{n}(n \geq 3)$}

Let i.i.d. random vectors $\left\{X_{i}\right\}_{i=1,2, \ldots, m}$ be uniformly distributed in the unit ball of $\mathbb{R}^{n}, n \geq 3$. In this section we study the probability that a ball with a small radius in $\mathbb{R}^{n}$ does not belong to the convex hull of $\left\{X_{i}\right\}_{i=1,2, \ldots, m}$. If we use a similar method as in Section 3, then new difficulties arise on taking into account too many different cases, and computing several complicated volumes, multiple integrals, etc. Instead of computing the exact value of the probability, we give non-trivial upper estimates of it in this section based on the idea used in Section 2. To this end, let $p^{\delta}(k, m)$ be the probability that the ball in $\mathbb{R}^{n}$ with radius $\delta$ does not belong to the convex hull of $\left\{X_{i}\right\}_{i=1,2, \ldots, m}$ which lie on a $k$-dimensional subspace of $\mathbb{R}^{n}$.

Theorem 4.1. Let $\left\{X_{i}\right\}_{i=1,2, \ldots, m}$ be independently and uniformly distributed random vectors in the unit ball of $\mathbb{R}^{n}, n \geq 3$, and $p^{\delta}(n, m)$ be the probability that a ball with a small radius $\delta$ in $\mathbb{R}^{n}$ does not belong to the convex hull of $\left\{X_{i}\right\}_{i=1,2, \ldots, m}$. It holds that $p^{\delta}(n, m) \leq p_{*}^{\delta}(n, m)$ where $p_{*}^{\delta}(n, m)$ solves

$$
\left\{\begin{array}{l}
p_{*}^{\delta}(n, m)=\frac{1}{2} p_{*}^{\delta}(n, m-1)+\frac{1}{2} p_{*}^{\delta}(n-1, m-1), \\
p_{*}^{\delta}(k, 1)=1-\delta^{k} \text { and } p_{*}^{\delta}(1, k)=\frac{(1-\delta)^{k}}{2^{k-1}}, \text { for } k \geq 1 .
\end{array}\right.
$$

In particular,

$$
\begin{aligned}
& p^{\delta}(n, m) \leq 1-\delta^{n+1-m}\left(\frac{1+\delta}{2}\right)^{m-1}, \text { for } m \leq n \\
& p^{\delta}(n, n+1) \leq 1-\frac{1}{2^{n}}\left[(1+\delta)^{n}+\delta-\delta^{2}\right] .
\end{aligned}
$$

Remark 4.1. We recall the probabilities $p(n, m)$ that the origin does not belong to the convex hull of $m$ random vectors in $\mathbb{R}^{n}$ discussed in Section 2. The probabilities are

$$
p(n, m)=2^{-m+1} \sum_{k=0}^{n-1}\left(\begin{array}{c}
m-1 \\
k
\end{array}\right) \text { for } n<m,
$$

and $p(n, m)=1$ for $n \geq m$. It is then obvious that a trivial upper bound of $p^{\delta}(n, m)$ is $p(n, m)$, that is $p^{\delta}(n, m) \leq p(n, m)$. This gives $p^{\delta}(n, m) \leq 1$ for $m \leq n$, and $p^{\delta}(n, n+1) \leq 1-\frac{1}{2^{n}}$. Thus the upper bounds in (4.2) and (4.3) are slightly better than these.

Proof of Theorem 4.1. Following the idea in the proof of Theorem 2.1 in Section 2, we will show

$$
p^{\delta}(n, m) \leq \frac{1}{2} p^{\delta}(n, m-1)+\frac{1}{2} p^{\delta}(n-1, m-1) .
$$

To this end, let $h$ be the indicator function of the event that the ball with radius $\delta$ is not in the convex hull of $\left\{X_{i}\right\}_{i=1,2, \ldots, m}$, and $\left\{\varepsilon_{i}\right\}$ be i.i.d. Bernoulli random variables. Then by the same reasoning in Section 2, we have, with $\varepsilon^{\prime}=\left(\varepsilon_{1}, \ldots, \varepsilon_{m-1}\right)$,

$$
\begin{aligned}
p^{\delta}(n, m)= & \mathbb{E}_{\varepsilon^{\prime}} \mathbb{E}_{X} \mathbb{E}_{\varepsilon_{m}} h\left(\varepsilon_{1} X_{1}, \ldots, \varepsilon_{m} X_{m}\right) \\
= & \frac{1}{2} \mathbb{E}_{\varepsilon^{\prime}} \mathbb{E}_{X} \mathbb{E}_{\varepsilon_{m}} h\left(\varepsilon_{1} X_{1}, \ldots, \varepsilon_{m-1} X_{m-1}\right) \\
& +\frac{1}{2} \mathbb{E}_{\varepsilon^{\prime}} \mathbb{E}_{X}\left[2 \mathbb{E}_{\varepsilon_{m}} h\left(\varepsilon_{1} X_{1}, \ldots, \varepsilon_{m} X_{m}\right)-h\left(\varepsilon_{1} X_{1}, \ldots, \varepsilon_{m-1} X_{m-1}\right)\right] \\
= & \frac{1}{2} p^{\delta}(n, m-1)+\frac{1}{2} \mathbb{E}_{\mathcal{\varepsilon}^{\prime}} \mathbb{E}_{X} R
\end{aligned}
$$


where the random variable $R \in\{0,1\}$ is

$$
R=h\left(\varepsilon_{1} X_{1}, \ldots, X_{m}\right)+h\left(\varepsilon_{1} X_{1}, \ldots,-X_{m}\right)-h\left(\varepsilon_{1} X_{1}, \ldots, \varepsilon_{m-1} X_{m-1}\right) .
$$

In Section 2, an equivalent statement of the event $R=1$ was found. But here we can only show

$$
\{R=1\} \subseteq\left\{h\left(\varepsilon_{1} X_{1}, \ldots, \varepsilon_{m-1} X_{m-1}\right)=1\right\}
$$

To see (4.5), we notice that when $h\left(\varepsilon_{1} X_{1}, \ldots, X_{m}\right)=h\left(\varepsilon_{1} X_{1}, \ldots,-X_{m}\right)=1$, then the orthogonal projection $u_{i}$ of $X_{i}$ onto $X_{m}^{\perp}, 1 \leq i \leq m-1$, should satisfy $h\left(\varepsilon_{1} u_{1}, \ldots, \varepsilon_{m-1} u_{m-1}\right)=1$. This is (4.5). Thus the probabilities $p^{\delta}(n, m)$ satisfy (4.4) with known boundary values $p^{\delta}(k, 1)=1-\delta^{k}$ and $p^{\delta}(1, k)=\frac{(1-\delta)^{k}}{2^{k-1}}$ for $k \geq 1$. Now we solve the corresponding difference equation (4.1). Obviously $p^{\delta}(n, m) \leq p_{*}^{\delta}(n, m)$ from comparisons. What is more, the equation (4.1) can be solved as

$$
\begin{aligned}
& p_{*}^{\delta}(n, m)=1-\delta^{n+1-m}\left(\frac{1+\delta}{2}\right)^{m-1}, \text { for } m \leq n \\
& p_{*}^{\delta}(n, n+1)=1-\frac{1}{2^{n}}\left[(1+\delta)^{n}+\delta-\delta^{2}\right] .
\end{aligned}
$$

Thus (4.2) and (4.3) are directly from (4.6). By inductions, it is also feasible to find general $p_{*}^{\delta}(n, m)$, which have more complicated expressions.

Acknowledgment. We are grateful to Professor Frank Gao for stimulating discussions and useful suggestions, to an anonymous referee for helpful comments.

\section{References}

[1] J. Bardsley, J. Nagy, Covariance-preconditioned iterative methods for nonnegatively constrained astronomical imaging, SIAM Journal on Matrix Analysis and Applications, 27, 4, 1184-1197, (2006)

[2] G. Baxter, A combinatorial lemma for complex numbers, The Annals of Mathematical Statistics, 32, 3, 901-904, (1961)

[3] D. Donoho, et al., Maximum entropy and the nearly black object, Journal of the Royal Statistical Society, 54, 1, 41-81, (1992)

[4] D. Donoho, J. Tanner. Counting the faces of randomly-projected hypercubes and orthants, with applications, Discrete \& Computational Geometry, 43, 3, 522-541, (2010)

[5] B. Efron, The convex hull of a random set of points, Biometrika, 52, 331-343, (1965)

[6] N. Jewell, J. Romano, Coverage problems and random convex hulls, Journal of Applied Probability, 19, 546-561, (1982)

[7] L. Li, T. Speed, Parametric deconvolution of positive spike trains, The Annals of Statistics, 28, 5, 1279-1301, (2000)

[8] A. Rényi, R. Sulanke, Über die konvexe Hülle von $n$ zufällig gewählten Punkten, Probability Theory and Related Fields, 2, 1, 75-84, (1963)

[9] S. Majumdar, A. Comtet, J. Randon-Furling, Random convex hulls and extreme value statistics, Journal of Statistical Physics, 138, 6, 955-1009, (2010)

[10] F. Spitzer, H. Widom, The circumference of a convex polygon, Proceedings of the American Mathematical Society, 12, 3, 506-509, (1961)

[11] J. Wendel, A problem in geometric probability, Mathematica Scandinavica, 11, 109-111, (1962) 